\title{
The forming technology and use of titanium alloy elbow made by diameter-expanding and pushing
}

\author{
CHEN JUN $^{1, a}$, DUAN WENSEN ${ }^{2, b}$ and YANG HAIYING ${ }^{3, c}$ \\ 1, 2, 3 Northwest institute for nonferrous metal research, Xi'an, Shaanxi, China \\ achristinchenjun@163.com, ${ }^{b}$ duanwensen@c-nin.com, c yanghaiying@sohu.com
}

Keywords:Elbow;titanium alloy;deforming technology of elbow; elbow made by diameter-expanding and pushing

\begin{abstract}
Titanium alloy has good corrosion resistance, so it has been used as pipeline for delivering corrosion media. The forming technologies of titanium alloy have many types, such as elbow made by diameter-expanding and pushing; elbow made by pressing plate and welding, elbow made by die pressing with mould, elbow made by casting and shrimp-waist elbow made by slicing cutting and welding et al. The titanium alloy elbow made by diameter-expanding and pushing has characteristic of uniform thickness, high production efficiency and no-welding joint et al, the paper introduces the forming principle of titanium alloy elbow made by diameter-expanding and pushing and service condition of titanium alloy.
\end{abstract}

\section{Introduction}

The elbow has very various forming technologies, which consists of elbow made by diameter-expanding and pushing, elbow made by extruding along tunnel, elbow made by roll depression along drop center wheel, shrimped-shaped elbow, elbow made by pressing plate and welding, elbow made by bending press with section mould, elbow made by casting et al ${ }^{[1,2]}$. Because titanium and titanium alloys have bad deformation plasticity in room temperature, high yield ration. The tensile strength of titanium alloys increases with temperature increasing, so the deformation temperature range of titanium alloy elbow is very narrow. In present, the used methods of titanium alloy elbow comprise the method of elbow made by diameter-expanding and pushing, shrimped-shaped elbow, elbow made by pressing plate and welding, elbow made by bending press with section mould, elbow made by casting.

Because the shrimp-shaped elbow is spliced by using seamless tube or roll-welded cylinder, it has high material cost, multiwelded joint, bad corrosion resistance, bad appearance and flexuous inner surface without smooth transition which leads to high transmission resistance and low service life.

As a result of casting formation, the wall thickness of elbow made by casting is thicker than that of pipeline, and results in difficulty matching with pipeline. The surface smoothness is very bad, and the defect such as gas holes exist in internal of material due to casting, and seriously causes corrosion resistance and pressure resistance.

Comparing with elbow made by pressing with section mould, elbow made by pressing plate and welding, elbow made by casting, the elbow made by diameter-expanding and pushing has an advantage of large range specification, homogeneous wall thickness, good surface smoothness, elegant appearance, high production efficiency, especially excellent corrosion resistance when used for transporting high corrosion mediator and high safe reliability. The technology of elbow made by diameter-expanding and pushing and its application on titanium alloy elbow is discussed in this paper.

\section{Elbow made by diameter-expanding and pushing [3]}

The dimension parameter of isometric elbow is illustrated in Fig.1. The outer diameter D and thickness $\mathrm{T}$ is everywhere equal, the bending radius is $\mathrm{R}$, the size from center line to end plane is 
equal to $\mathrm{R}$, and the nominal diameter is a standardization parameter. The standardization elbow is divided into two types elbow according to the ratio of $\mathrm{R}$ to $\mathrm{Dn}$. The long diameter elbow is 1.5 , and that the short diameter elbow is 1.0. Because the amplititude of expanding diameter and bending of short radius elbow is larger than that of long radius elbow, the forming technology of short radius elbow is more difficulty than that of long radius elbow.

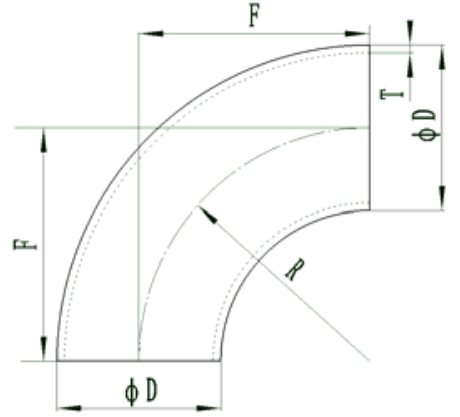

Fig.1 Schematic illustration of isometric elbow

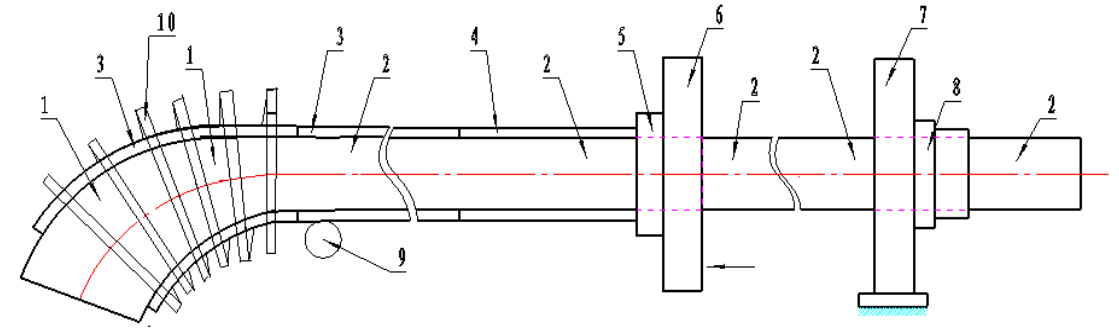

1. Mandrel 2.Mandrel bar 3.Elbow 4.Pipe 5.Pushing hoop 6.Movalble frame 7.Fixing frame 8.Supporting roller wheel 9.Supporting roller wheel 10 . Heating coil

Fig.2 Schematic illustration of pushing bends by expanding diameter

Processing schematic illustration of isometric elbow made by expanding and bending is shown in Fig.2. During diameter-expanding and bending of elbow, the pipe covers on core bar, and the core bar is fixed on framework. The movable frameworks push forward pushing ring, pushing pipe and pipes along core bar. When the pipes enter into the range of mandrel, they are heated to a degree temperature by medium frequency induction heating, and they generate expanding diameter along circumference and bending along axial direction, and finally become elbow after getting over core bar.

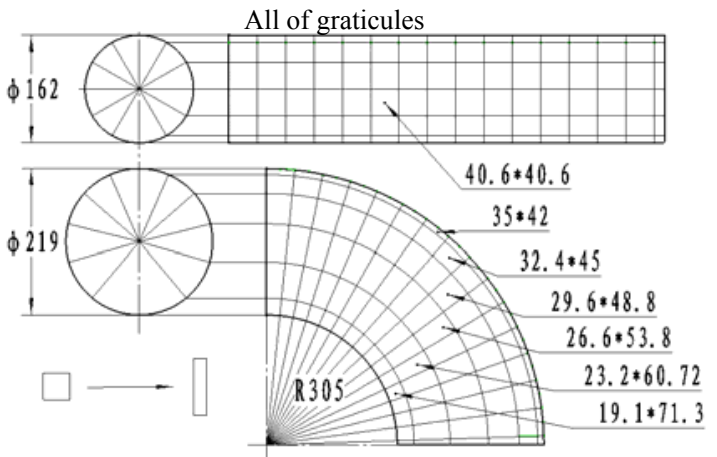

The griddings on inner arc

Fig. 3 the gridding change during pushing bend by expanding diameter ${ }^{[6]}$

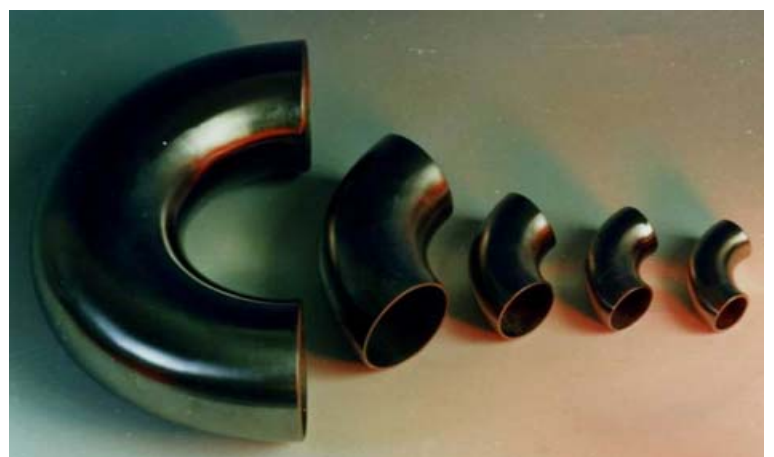

Fig.4 Seamless titanium elbows

The principle of elbow made by diameter-expanding and bending is that the outer arc basically don't exists deformation, however the inner arc generates compression along axial direction and expansion along circumference, the wall thickness on inner arc has no change. In general, the elbow made by diameter-expanding and pushing is proportion deformation ${ }^{[4.5]}$. The surface of pipe is divided into many small equivalence graticules. After diameter-expanding and pushing, the graticules on outer arc of elbow became rectangle from square, whereas the graticules on inner arc of elbow take place no change. From inner arc to outer arc, the deformation degree of graticules gradually decreases, and the ratio of height to width also reduces (shown in Fig.3 ) ${ }^{[6]}$.During diameter-expanding and pushing of elbow, the pipe is subjected to tension deformation and compression deformation ${ }^{[7]}$. The method of elbow made by diameter-expanding and pushing can avoid non-uniform phenomenon of wall thickness comparing with conventional bending elbow technology, because the latter method causes reduction of wall thickness on outer arc because of tension deformation and increase of wall thickness on inner arc due to compression deformation. 
The key of elbow made by diameter-expanding and pushing is design and fabrication of mandrel and careful control of technology parameters. The technology parameters of elbow made by diameter-expanding and pushing are involved with design and fabrication of heating coil, position of heating coil, medium frequency, pipe heating temperature, distribution of temperature, pushing velocity, pipe size, mandrel smoothness, choice of lubrication and using method of lubrication et al.

The elbow made by diameter-expanding and pushing can assure outer diameter roundness and wall thickness uniformity of elbow. The design of mandrel is prone to using mathematic curve as centre line of mandrel and adopting elliptical cross section structure ${ }^{[8]}$. These design method can ensure elbow made by diameter-expanding and pushing not to be subjected reshaping after primary forming, greatly simplify production procedure, increase production efficiency and ensure product quality. This elbow method is suitable for various elbows which diameter is from 32 to 1020 millimeter with various turn angles such as $30^{\circ} 、 45^{\circ} 、 60^{\circ} 、 90^{\circ} 、 180^{\circ}$ and $270^{\circ}$. The elbows include non-seam elbow made by non-seam pipe and single welded seam elbow produced by roll-welded cylinder. Because the stress condition on inner arc of elbow is more formidable than that on outer arc and side arc, the welded-seam is generally placed on outer arc or side arc of elbow during diameter-expanding and pushing. The elbows made by diameter-expanding and pushing have advantage of good shape, uniform wall thickness and same wall thickness comparing with wall thickness of pipeline.

Because titanium alloys have bad deformation plasticity in room temperature, high yield ratio. The tensile strength of titanium alloys increases with temperature increasing, so the deformation temperature range of titanium alloy elbow is very narrow. Furthermore, titanium alloy easily oxidizes at high temperature owing to high chemical activity, and easily generates bonding with steel core. Therefore, the measurement of antioxidizaion and antibonding should be adopted. The titanium alloy elbow should be controlled deformation temperature and adopted effective surface protection measurement ${ }^{[9]}$.

The mass production of elbow and pipe fittings of titanium alloy in China was commenced in the late of 1990. Northwest institute for non-ferrous metal research began using method of diameter-expanding and pushing to produce seamless elbow or single seam elbow, and started using method of pressing plate and welding to manufacture longitudinal welded seam elbow. A series titanium alloy elbows were produced until 1992, included seamless elbow which was less than diameter $325 \mathrm{~mm}$ and seam elbow with longitude welded joint which was more than diameter $325 \mathrm{~mm}$. These elbows can achieve requirement of steel products according to national standardization GB12459-90. Seamless titanium elbows are shown in Fig.4.

\section{Discussion}

Standards of titanium alloy elbow.After many times modified, the finally version standard of titanium pipe fittings in American was edited in 1995, such as ASTM 363-1995 Standard Specification for Seamless and Welded Unalloyed Titanium and Titanium Alloy Welding Fittings and ASME SEC II-B SB363-1995 Specification for seamless and Welded Unalloyed Titanium and Titanium Alloy Welding Fittings ASTM B363-93 ( Boiler and Pressure Vessel Codes). The specifies size, shape and tolerance of pipe fittings in ASTM 363-1995 are equal to a series standards such as ASTM/ANSI B16.9-1986 Factory-Made Wrought steel Butt welding Fittings, ANSI SP-43 Factory-Made Wrought steel Butt-welding Fittings), ANSI/ASME B36.19M-1986 Stainless Steel Pipe $^{[10]}$.

As the mass production and order grow, Northwest institute for Non-ferrous metal research in succession institutes enterprise standards and these works settle good basis of drawing up standard for titanium pipe fitting. In order to instruct standardization production, design and using, purchasing and sailing, installment and construction of titanium alloy elbow, Chinese national petroleum and chemical industry parts issue profession standard HG/T 3651-1999 unalloyed titanium and titanium alloy butt-welding seamless pipe fittings ${ }^{[12]}$. 
In present, Northwest institute for nonferrous metal research is only production factory for producing pipe fittings of titanium, zirconium, nickel used in large diameter seamless pipe line and extra-high wall thickness pipeline in china, and is a fixed production factory for producing pipeline fitting part of titanium and mare metal in chemical engineering department. It can product many angle elbows including $45^{\circ}, 90^{\circ}, 180^{\circ}$ in keeping with Chinese standard and chemical engineering standard. Now, the elbow of titanium alloy is indispensable standardization parts and components in corrosion resistance pipeline engineering of petroleum, chemical engineering, metallurgy, electric power and producing salt field ${ }^{[11]}$.

Application of titanium made by diameter- expanding and pushing. Owing to the appearance and using of standard titanium and its alloys elbow, the integral life of pipeline increases, the shutdown cost and maintenance cost decreases. The titanium elbow is suitable to deliver $\mathrm{NaCl}$ solution, wet chlorine, organic acid anhydride et al no-room temperature weakly acidic medium, salt of high corrosion and organic acid medium. So the titanium elbow made by diameter-expanding and pushing is used in ion-exchange membrane electrolyzer in chemical engineering factory and effectively settle percentage of home-made parts of titanium alloy ${ }^{[1]}$.

Closed air collector of titanium is a special apart used in aviation and space field, and ring pipe is main body of ring-shaped air collector. Northwest institute for non-ferrous metal research produced ring pipe of $\alpha$-type pure titanium and $\beta$-type Ti-26 turn angle titanium alloy with more than $270^{\circ}$ made by diameter-expanding and pushing in the late of 1990 . The method can settle the shortcoming of ring air collector made by made by pressing plate and welding with two long $360^{\circ}$-longitudinal welded seams, which was a weak link in pressure container. Father more, the elbow made by pressing plate and welding can not assure same wall thickness, and exists problems such as high fabrication cost, complex technology and low rate of finished products ${ }^{[13]}$.

With the successful application of finite element simulation in the processing of material, the material researchers have carried out large finite element simulations on titanium elbow and ring-shaped air collector made by diameter-expanding and pushing. Zhou Wei et al proposed the design method of ram's core bar axis using Archimedes' screw, and carried out finite element simulation on uniformity of wall thickness and stress distribution on inner arc and outer arc during deformation of elbow. The change of equivalent stress value increased with the increasing of bending angle, the stress on inner arc was higher than that on outer arc during diameter-expanding and pushing of elbow. The tress distribution showed that the inner arc suffered compression stress which affected in the increasing of wall thickness, and the outer arc bearded tensile stress which leaded to the decrease of wall thickness ${ }^{117]}$. Li Lintao studied the effect of Archimedes' parameter in design of sam core bar and pushing velocity on deformation procession of ring-shaped elbow made by diameter- expanding and pushing in order to optimize processing parameter. He suggested the wall thickness of elbow was uniformity when Archimedes' screw coefficient was 1.1; pushing velocity was $4.0 \mathrm{~m} / \mathrm{s}$ during deformation of ring-shaped elbow ${ }^{[18]}$. Yin Jin-ou researched the influence of various size graticule elements on deformation processing of ring-shaped elbow made by diameter-expanding and pushing. He suggested single graticule element was suitable to simulate the manufacturing procedure of small diameter elbow made by diameter-expanding and pushing. However, the division of single graticule element was not fit for simulation of large diameter elbow, and resulted in difficulty of simulation, even simulation failure. The assembly of various size graticule elements can reduce repetitive computation number and successfully assure accomplishment of simulation ${ }^{] 19]}$. Tang Suanbing speculated the stress and strain distribution on outer arc and inner arc and failure strength of ring-shaped air collector of near $\alpha$ titanium alloy Ti-3Al-2.5V and $\beta$ type titanium alloy Ti26. The strength resistance of $\beta$ type titanium alloy was superior to that of near $\alpha$ type titanium alloy. The maximum strength located on inner arc of ring-shaped air collector, which leaded to initiating point of burst ${ }^{120]}$.

Outlook.As Chinese processing technology of titanium alloys and production capacity of equipment grow, the large diameter pipe and wide plate of titanium alloy are produced by special processing technologies. These materials provide advantage condition to produce titanium non-seam 
elbow or single- welded elbow made by diameter-expanding and pushing and to promote the specification of titanium alloy elbow. Titanium alloy elbow is widely used in shipbuilding, petroleum, and chemical engineering fields.

\section{Conclusion}

1) The titanium alloy elbow made by diameter-expanding and pushing has an advantage over other production methods of elbow. The titanium alloy elbow possesses good exterior appearance, smoothness surface, homogeneous wall thickness, effectively prevent corrosion and highly increase using life.

2) The production of titanium elbow made by diameter-expanding and pushing promotes the formulation of titanium elbow standard, and accelerates unprecedented development of design, production and using of titanium elbow.

\section{References}

[1] Duan Wensen. Acta Metallurgica Sinica, 2002, 38(sup.1): 422 424

[2] Dai Hongwei, Duan Wensen. Titanium industry progress[J], 1999,(1):30-33

[3] Jiang Zhengyi, Zhao Xianglai. Journal of plasticity engineering, 1997,4(4):65 69

[4] Lu Xiaoyang, Lu Xiaoli. Metal forming machiner, 1997, (4):10-13

[5] Lu Xiaoyang, Xu Bingye ,Cen Zhangzhi,Shi Baojun. Mechanics and engineering, 1998,20(6):15-17

[6] Duan Wensenet al. 1st Symposia Proceedings of Chinese pipeline acdamic,. Hefei: Chinese pressure vessel association pipeline committee, 1997: 79-83

[7] Li Kui. Construction technology (Ji Lin). 1994, (2):32-38

[8] Ma Li. Pipeline Technique and Equipment, 1998,(4):18-22

[9] Duan Wensen.. Acta Metallurgica Sinica, 2002,38(sup.1):422 424

[10] Duan Wensen. Pipeline Technique and Equipment, 2001, (6):20-22

[11] Duan Wensen. Titanium industry process, 1993,(3): 54-56

[12] Zhaoguorui. Titanium industry progress [J], 1995, (1): 28-29

[13] Duan Wensen, Li Danke, DuYu, Li Wei, Guo Hongchao. Rare metal materials and engineering, 2005,34(Supp1.3): 504-507

[14] Yang Yingli, Duan Wensenet al.China patent 02101193.1 (2002)

[15] Yang Yingli, Yang Junet al. China patent 2004100087296.1（2004）

[16] Tian Jin, Wu En. Rare metal materials and engineering, 2005,34(Suppl.3):733-735

[17] Zhou Wei, Zhou Lian er al. Rare metal materials and engineering, 2005, 34 (10):1585-1587

[18] Li Lintao, ZENG Weidong et al. Forging \& Stamping echnology, 2006, 31 (6) : 131-134

[19]Yin Jin-ou, Yang Yinli, Zhao Hengzhang et al. RARE METAL MATERIALS AND

ENGERING, 2005,34(Suppl.3): 622-624

[20]Tan Suanbin, Duan Wensen et al. Rare metal materials and engineering, 2005, 34(Suppl.3):690-693 